\title{
Overexpression of DJ-1 reduces oxidative stress and attenuates hypoxia/reoxygenation injury in NRK-52E cells exposed to high glucose
}

\author{
ZI-YING SHEN $^{1 *}$, QIAN SUN ${ }^{2 *}$, ZHONG-YUAN XIA ${ }^{2}$, QING-TAO MENG $^{2}$, SHAO-QING LEI ${ }^{2}$, \\ BO ZHAO ${ }^{2}$, LING-HUA TANG ${ }^{2}$, RUI XUE ${ }^{2}$ and RONG CHEN ${ }^{2}$ \\ ${ }^{1}$ Department of Anesthesiology, Affiliated Hospital of Qingdao University Medical College, Qingdao, Shandong 266003; \\ ${ }^{2}$ Department of Anesthesiology, Renmin Hospital of Wuhan University, Wuhan, Hubei 430060, P.R. China
}

Received October 12, 2015; Accepted June 30, 2016

DOI: $10.3892 / \mathrm{ijmm} .2016 .2680$

\begin{abstract}
Patients with diabetes are more vulnerable to renal ischemia/reperfusion ( $\mathrm{I} / \mathrm{R})$ injury, which is implicated in hyperglycemia-induced oxidative stress. We previously reported that the hyperglycemia-induced inhibition of DJ-1, a novel oncogene that exhibits potent antioxidant activity, is implicated in the severity of myocardial I/R injury. In the present study, we aimed to explore the role of DJ-1 in hypoxia/reoxygenation (H/R) injury in renal cells exposed to high glucose (HG). For this purpose, NRK-52E cells were exposed to $\mathrm{HG}(30 \mathrm{mM})$ for $48 \mathrm{~h}$ and then exposed to hypoxia for $4 \mathrm{~h}$ and reoxygenation for $2 \mathrm{~h}$, which significantly decreased cell viability and superoxide dismutase (SOD) activity, and increased the malondialdehyde (MDA) content, accompanied by a decrease in DJ-1 protein expression. The overexpression of DJ-1 by transfection with a DJ-1 overexpression plasmid exerted protective effects against HG-induced H/R injury, as evidenced by increased CCK-8 levels and SOD activity, the decreased release of lactate dehydrogenase (LDH) and the decreased MDA content, and increased nuclear factor (erythroid-derived 2)-like 2 (Nrf2) and heme oxygenase-1 (HO-1) expression. Similar effects were observed following treatment with the antioxidant, $\mathrm{N}$-acetylcysteine. These results suggest that the overexpression of DJ-1 reduces oxidative stress and attenuates $H / R$ injury in NRK-52E cells exposed to HG.
\end{abstract}

\section{Introduction}

Diabetes mellitus (DM) has gained increasing attention globally. Diabetic nephropathy (DN) is one of the most

Correspondence to: Professor Zhong-Yuan Xia, Department of Anesthesiology, Renmin Hospital of Wuhan University, 238 Jiefang Road, Wuhan, Hubei 430060, P.R. China

E-mail: xiazhongyuan2005@aliyun.com

*Contributed equally

Key words: DJ-1, high glucose, hypoxia/reoxygenation injury, oxidative stress, NRK-52E cells seriousdiabetic microvascular complications in DM, which leads to end-stage renal failure, seriously threatening the lives of patients (1). Recent studies have shown that oxidative stress plays an important role in the progression of $\mathrm{DN}(2,3)$. Ischemic injury often occurs in patients with diabetes during the peri-operative period. Diabetic nephropathy with ischemia/ reperfusion (I/R) injury is also closely related to oxidative stress $(4,5)$. However, the underlying mechanisms responsible for the adverse effects caused by oxidative stress on renal injury induced by hyperglycemia with $\mathrm{I} / \mathrm{R}$ insults have not yet been completely elucidated.

Currently, several hypotheses have emerged regarding DJ-1, which is also known as Park 7. DJ-1 was originally described as an oncogene (6). It is a multifunctional protein related to Parkinson's disease, neurodegeneration and oxidative stress $(7,8)$. Several lines of evidence have demonstrated the antioxidative function of DJ-1 in various disease models both in vitro and in vivo (9-12). In a study on hypertensive nephropathy, the physiological role of DJ-1 was shown to be associated with reactive oxygen species (ROS) in primary renal tubular epithelial cells (13). Another study demonstrated a compensatory increase in DJ-1 expression in the renal cortex against increased oxidative stress of the hyperglycemic milieu (14). Thus, it is suggested that DJ-1 may be effective against oxidative stress and it is increasingly considered as an important target for DN therapy (15-17).

The protective role of DJ-1 has been indicated in many organs and tissues, such as the heart, brain, liver, kidneys and pancreas. It has been shown that DJ-1 exerts neuroprotective effects against ischemic damage to the spinal cord through its antioxidant functions (18). The overexpression of DJ-1 may participate in a protective strategy against I/R injuryinduced oxidative stress in rat heart-derived H9c2 cells (19). We previously demonstrated that the hyperglycemia-induced inhibition of DJ-1 expression was implicated in the severity of myocardial I/R injury (20). However, the potential mechanisms of action of DJ-1 in renal cells exposed to high glucose (HG) and hypoxia/reoxygenation $(\mathrm{H} / \mathrm{R})$ injury have not yet been fully clarified. Moreover, DJ-1 can regulate the expression of various antioxidant genes, including nuclear factor (erythroidderived 2)-like 2 (Nrf2) $(21,22)$ and heme oxygenase-1 (HO-1), enhancing the antioxidant ability of cells (23). 
In the present study, we hypothesized that the overexpression of DJ-1 reduced oxidative stress and attenuated H/R injury in rat proximal tubular epithelial (NRK-52E) cells exposed to HG. As the antioxidant, $\mathrm{N}$-acetylcysteine (NAC), has been shown to protect the kidneys against $\mathrm{I} / \mathrm{R}$ injury by regulating the Nrf2 signaling pathway (24), we therefore, also examined the effects of NAC and compared them to those of DJ-1.

\section{Materials and methods}

Materials. The following materials were used: NRK-52E cells (American Tissue Type Culture Collection, Manassas, VA, USA); Dulbecco's modified Eagle's medium (DMEM; HyClone, Logan, UT, USA); phosphate-buffered saline (PBS; Gino Biological Medical Technology Co., Ltd., Hangzhou, China); fetal bovine serum (FBS; Gibco, Carlsbad, CA, USA); penicillin and streptomycin (Beyotime Institute of Biotechnology, Haimen, China); $0.25 \%$ Trypsin with $0.02 \%$ ethylenediaminetetraacetic acid (EDTA) (Gino Biological Medical Technology Co., Ltd.); D-Glucose (Sinopharm Chemical Reagent Co., Ltd., Shanghai, China); NAC (Sigma, St. Louis, MO, USA); the empty vector plasmid and pEX-2-EGFP-DJ-1 (GenePharma, Suzhou, China); Attractene transfection reagent (Qiagen, Valencia, CA, USA). The following kits were also used: the cell counting kit-8 (CCK-8; Dojindo, Kumamoto, Japan); lactate dehydrogenase (LDH) kit; superoxide dismutase (SOD) and malondialdehyde (MDA) kit (both from Nanjing Jiancheng Bioengineering Institute, Nanjing, China); the BCA protein assay kit; nuclear and cytoplasmic protein extraction kit (both from Beyotime Institute of Biotechnology). The antibodies used are listed as follows: rabbit anti-rat DJ-1 monoclonal antibody (\#5933; Cell Signaling Technology, Danvers, MA, USA); rabbit anti-rat Nrf2 (sc-722) and HO-1 (sc-10789) polyclonal antibody (Santa Cruz Biotechnology, Inc., Santa Cruz, CA, USA) rabbit anti-rat $\beta$-actin polyclonal antibody (GB13001; Wuhan Goodbio Technology Co., Ltd., Wuhan, China) and rabbit anti-rat Lamin B1 polyclonal antibody (BA1228; Boster, Wuhan, China). All other chemicals were obtained from commercial sources and were of highest grade available.

Cell culture. Rat proximal tubular epithelial (NRK-52E) cells were maintained in low-glucose DMEM medium (the concentration of glucose was $5.5 \mathrm{mM}$ ), which was supplemented with $10 \% \mathrm{FBS}, 100 \mathrm{U} / \mathrm{ml}$ penicillin and $0.1 \mathrm{mg} / \mathrm{ml}$ streptomycin, and the medium was replaced every $24 \mathrm{~h}$. The cells were subcultured using $0.25 \%$ trypsin with $0.02 \%$ EDTA after being washed with PBS twice and allowed to grow up to 70-80\% confluency.

The NRK-52E cells were inoculated in 6-well plates with lowglucose DMEM medium synchronously. When the cells grew to the appropriate density, they were incubated in low-glucose DMEM medium without FBS for $24 \mathrm{~h}$, pre-treated for $2 \mathrm{~h}$ with NAC (1 mM), incubated with low glucose concentrations (LG; final concentration, $5.5 \mathrm{mM}$ ) and $\mathrm{HG}$ (final concentration, $30 \mathrm{mM}$ ) for various periods of time, srespectively, and then exposed to hypoxia $\left(5 \% \mathrm{CO}_{2}, 1 \% \mathrm{O}_{2}\right.$ and $\left.94 \% \mathrm{~N}_{2}\right)$ for $4 \mathrm{~h}$, then to reoxygenation $\left(5 \% \mathrm{CO}_{2}, 21 \% \mathrm{O}_{2}\right.$ and $\left.74 \% \mathrm{~N}_{2}\right)$ for $2 \mathrm{~h}$.

Plasmid transfection. The day prior to transfection, the cells were seeded in 6-well plates at $30-50 \%$ confluence containing
$1 \%$ serum, $100 \mathrm{U} / \mathrm{ml}$ penicillin and $0.1 \mathrm{mg} / \mathrm{ml}$ streptomycin. The cells were $40-80 \%$ confluent on the day of transfection and were transfected with the plasmids according to the manufacturer's instructions. The empty vector plasmid and pEX-2-EGFP-DJ-1 $(4 \mu \mathrm{g})$ were transfected into the cells without FBS and removed after $12 \mathrm{~h}$ with fresh low-glucose and HG medium added respectively, and then exposed to hypoxia for $4 \mathrm{~h}$, then to reoxygenation for $2 \mathrm{~h}$.

Determination of cell viability and LDH assay. The cell suspension $(100 \mu \mathrm{l})$ was inoculated in 96-well plates, and the supernatant was collected for the LDH toxicity emitting experiment. According to the instructions of the manufacturer of the LDH kit, the absorbance was measured at $450 \mathrm{~nm}$ using a microplate reader (Victor3 1420-050; Perkin Elmer, Waltham, MA, USA). The medium was removed and the cells were washed twice with PBS. The fresh medium and $10 \mu \mathrm{l} \mathrm{CCK-8}$ solution were added to each well followed by incubation for $2 \mathrm{~h}$ in $37^{\circ} \mathrm{C}$ and $5 \% \mathrm{CO}_{2}$. The absorbance was then measured at $450 \mathrm{~nm}$ using a microplate reader (Victor3 1420-050; Perkin Elmer) and the cell viability was calculated.

Detection of oxidative stress. The NRK-52E cells were washed 3 times with PBS and sonicated using an ultrasonic crusher (FB120220; Thermo Fisher Scientific, Waltham, MA, USA). The cells were examined to determine the contents of SOD and MDA using the respective kits (from Nanjing Jiancheng Bioengineering Institute) according to the manufacturer's instructions. Cellular protein was measured using the BCA protein assay kit. The MDA content in the NRK-52E cells was measured by the colorimetric method (MDA kit; Nanjing Jiancheng Bioengineering Institute). The absorbance $(530 \mathrm{~nm})$ was measured using a microplate reader (Victor3 1420-050; Perkin Elmer) and the results were expressed in nmol of MDA/ mg protein. SOD activity in the NRK-52E cells was measured at an optical density at $450 \mathrm{~nm}$ according to the WST-1 method (SOD kit; Nanjing Jiancheng Bioengineering Institute). The results were expressed as $\mathrm{U} / \mathrm{mg}$ protein and 1 unit of enzyme is defined as the enzyme activity that inhibits the autoxidation of pyrogallol by $50 \%$.

Western blot analysis. The cells were washed 3 times with PBS and trypsinized after almost completely covering the bottom of the wells. Cell suspensions were centrifuged for $5 \mathrm{~min}$ at $1,000 \mathrm{rpm}$, after which the supernatant was discarded. Total cellular proteins were extracted using RIPA lysis buffer (Beyotime Institute of Biotechnology). Nuclear and cytoplasmic proteins were extracted according to the manufacturer's instructions (nuclear and cytoplasmic protein extraction kit; Beyotime Institute of Biotechnology). An equal amount of protein was loaded onto sodium dodecyl sulfate (SDS) polyacrylamide gels, electrophoresed and transferred to PVDF membranes. To prevent non-specific background binding of the antibody, the membranes were blocked by using 5\% BSA and incubated for $2 \mathrm{~h}$ at room temperature under agitation. The membranes were then incubated with the specific rabbit anti-rat DJ-1 $(1: 1,000)$, Nrf2 (1:200), HO-1 (1:200), $\beta$-actin $(1: 2,000)$ and Lamin B1 (1:200) antibodies overnight at $4^{\circ} \mathrm{C}$. After repeated washing, the membranes were incubated with the corresponding goat anti-rabbit horseradish peroxidase-conjugated secondary 

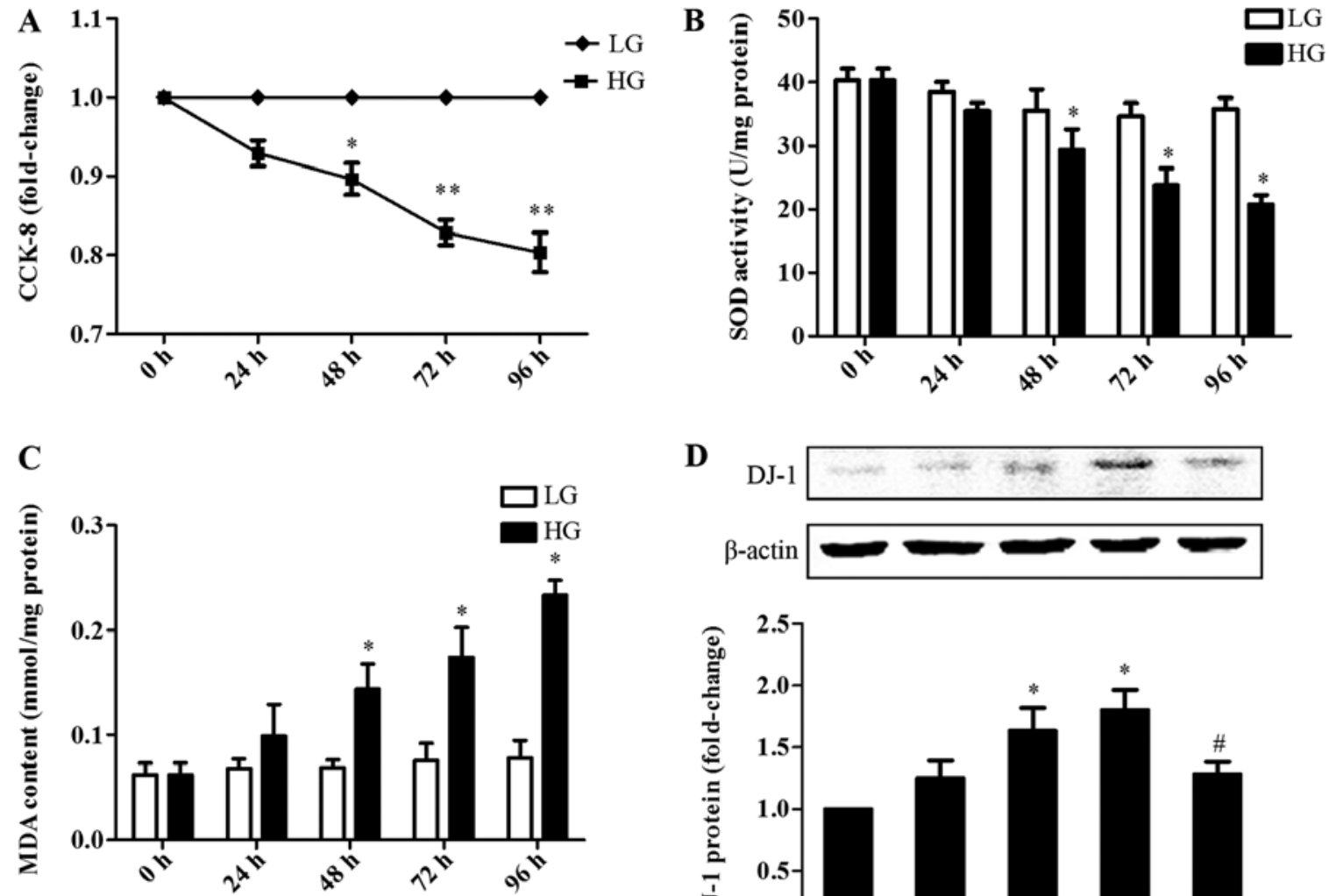

D
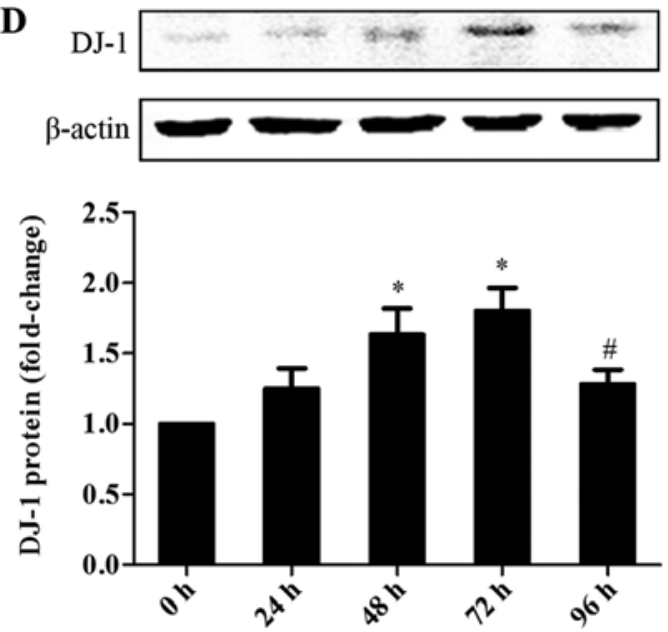

Figure 1. Effects of high glucose (HG) on the viability of, and oxidative stress and DJ-1 expression in NRK-52E cells over time. NRK-52E cells were exposed to HG and we then examined (A) cell viability by CCK-8 assay; (B) SOD activity; (C) the MDA content; (D) The relative protein level of DJ-1 was measured by western blot analysis. $\beta$-actin was used for normalization of the data. Data are expressed as the means $\pm \mathrm{SEM}$. ${ }^{*} \mathrm{P}<0.05,{ }^{* * *} \mathrm{P}<0.01$ compared with the 0 -h group; ${ }^{\text {"P }} \mathrm{P}<0.05$ compared with the 72 -h HG group. Results shown are representative of at least 3 independent experiments.

antibody (1:10,000; IRDye 800CW, LI-COR Biosciences, Lincoln, NE, USA) for $1 \mathrm{~h}$ at room temperature. The intensity of the identified bands accomplished with chemiluminescence was detected on an Odyssey two-color infrared laser imaging system (Li-Cor, Lincoln, NE USA) and densitometry was carried out using Odyssey software.

Statistical analysis. All data are presented as the means \pm SEM. All statistical analyses were performed using GraphPad Prism 5.0 software (GraphPad Software, La Jolla, CA, USA). Comparisons between multiple groups were evaluated by one-way analysis of variance (ANOVA) and comparisons between 2 groups by the Student's unpaired t-test. A value of $\mathrm{P}<0.05$ was considered to indicate a statistically significant difference.

\section{Results}

Effects of HG on cell viability, oxidative stress and DJ-1 expression in NRK-52E cells over time. We first examined the effects of $\mathrm{HG}$ on cell viability and oxidative injury in NRK-52E cells over time. Both cell viability measured by CCK- 8 assay and the antioxidant activity measured by SOD activity assay in the NRK-52E cells were significantly decreased following $48 \mathrm{~h}$ of exposure to $\mathrm{HG}$, with a decreasing tendency continuing $72-96 \mathrm{~h}$ after initial the HG exposure (Fig. 1A and B). By contrast, the
MDA content was significantly increased at $48 \mathrm{~h}$, and further increased 72-96 h following exposure to HG (Fig. 1C).

To investigate the role of DJ-1 in NRK-52E cells exposed to $\mathrm{HG}$, we also measured the protein expression of DJ-1. DJ-1 expression did not significantly increase until $48 \mathrm{~h}$, a peak increase occurred at $72 \mathrm{~h}$, and DJ-1 expression was reduced to basal levels within $96 \mathrm{~h}$ following exposure to HG (Fig. 1D). Thus, we selected the duration of exposure to HG to be $48 \mathrm{~h}$ for our subsequent experiments. The osmotic control, mannitol, exerted no effects on cell viability, injury and DJ-1 expression (data not shown).

Effects of H/R on cell viability, oxidative stress and DJ-1 expression in NRK-52E cells exposed to $H G$. As the diabetic kidneys are more vulnerable to I/R injury $(25,26)$, we thus wished to examine the effects of H/R on NRK-52E cells following exposure to HG. H/R significantly decreased the levels of CCK-8 and SOD activity as compared to the LG control or HG control groups not subjected to H/R (Fig. 2A and B). Following exposure to $\mathrm{HG}$ for $48 \mathrm{~h}, \mathrm{H} / \mathrm{R}$ further decreased the levels of CCK-8 and SOD activity $(\mathrm{P}<0.05, \mathrm{LG}+\mathrm{H} / \mathrm{R}$ vs. $\mathrm{HG}+\mathrm{H} / \mathrm{R})$. By contrast, the MDA content in the $\mathrm{HG}$ control group was much higher than that in the LG control group. H/R further increased the MDA content as compared with that in the LG control or HG control groups not subjected to H/R (Fig. 2C). Of note, 

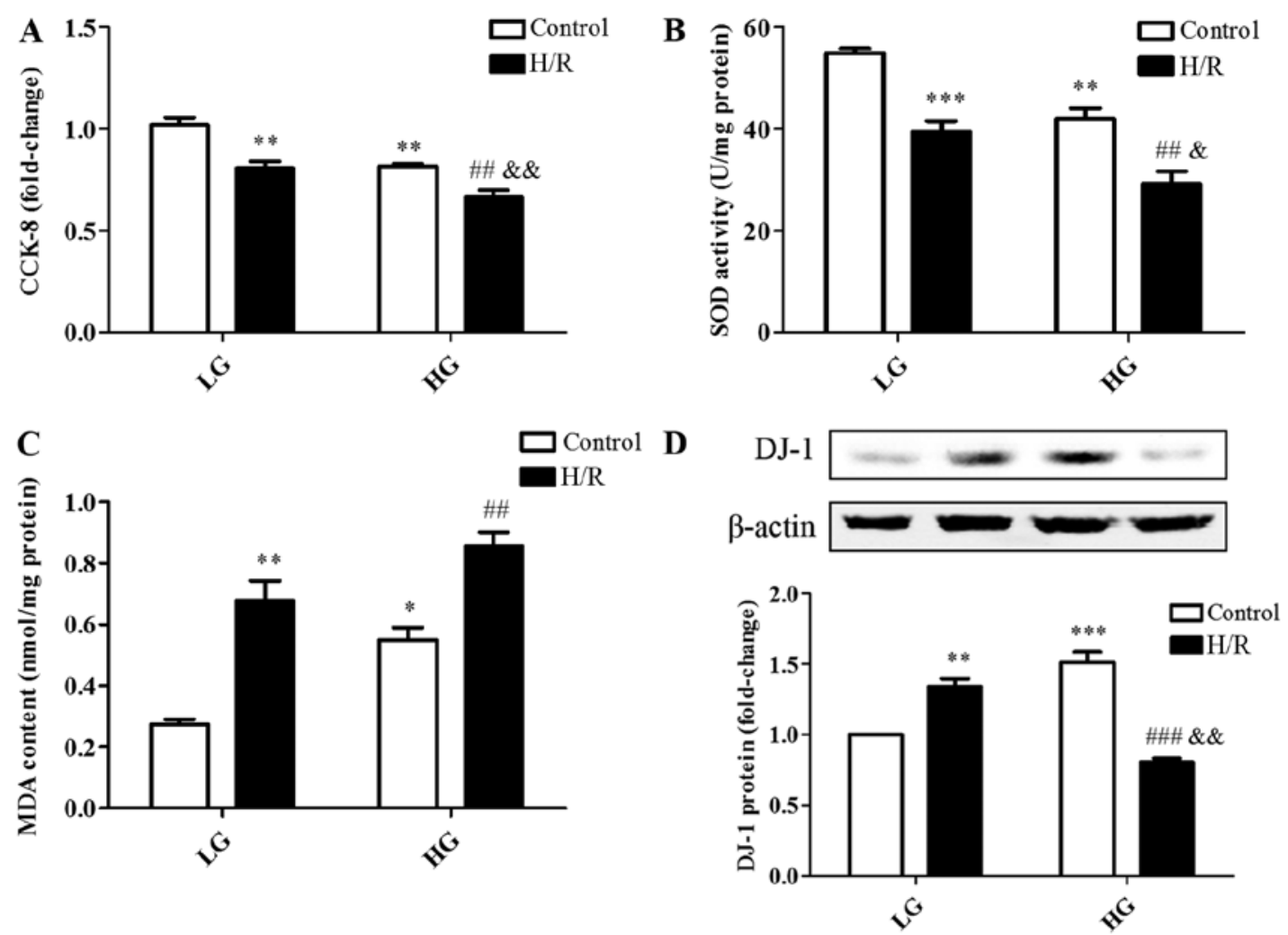

Figure 2. Effects of hypoxia/reoxygenation (H/R) on the viability of, and oxidative stress and DJ-1 expression in NRK-52E cells exposed to high glucose (HG). NRK-52E cells were exposed to HG and H/R and we then examined (A) cell viability by CCK-8 assay; (B) SOD activity; (C) the MDA content; (D) the relative protein level of DJ-1 was measured by western blot analysis. $\beta$-actin was used for normalization of the data. Data are expressed as the means \pm SEM. $P<0.05$, ${ }^{* *} \mathrm{P}<0.01,{ }^{* * *} \mathrm{P}<0.001$ compared with the LG control group; ${ }^{\# \#} \mathrm{P}<0.01$ compared with the HG control group; ${ }^{\mathrm{P}} \mathrm{P}<0.05$, \&\& $\mathrm{P}<0.01$ compared with the $\mathrm{LG}+\mathrm{H} / \mathrm{R}$ group. Results shown are representative of at least 3 independent experiments.

A

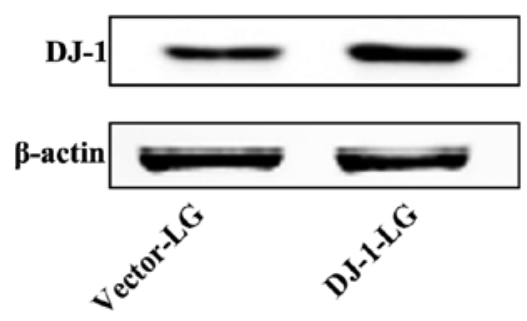

B

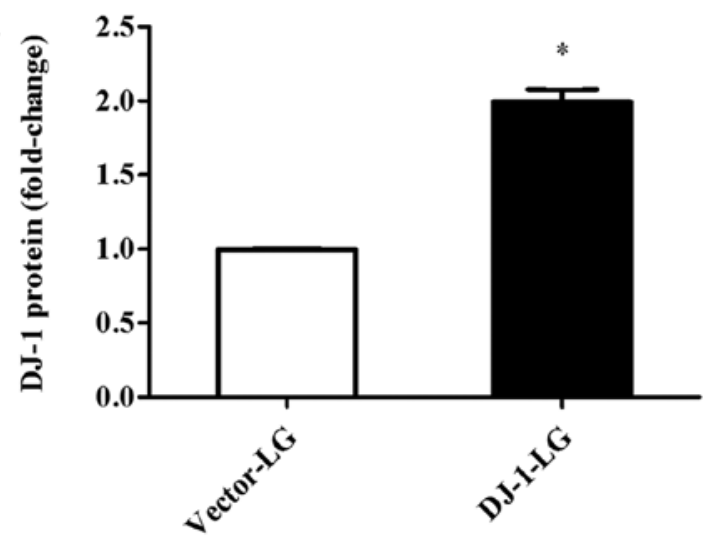

Figure 3. Overexpression of DJ-1 in the NRK-52E cells. (A) Western blot analysis was used to measure the protein expression of DJ-1 in cells transfected with pEX-2-EGFP-DJ-1. $\beta$-actin was used for normalization of the data. (B) Quantitative analysis of the relative protein level of DJ-1. Data are expressed as the means \pm SEM. ${ }^{*} \mathrm{P}<0.05$ compared with the vector-LG group (emtpy vector group). Results shown are representative of at least 3 independent experiments.

H/R significantly increased DJ-1 protein expression in the LG control group, but decreased DJ-1 protein expression in the NRK-52E cells following exposure to HG for $48 \mathrm{~h}$ (Fig. 2D). Thus, the decreased expression of DJ-1 may be involved in the vulnerability to renal I/R injury in diabetes.

Overexpression of DJ-1 in NRK-52E cells. To confirm the role of DJ-1 in H/R injury in renal cells, we transfected the NRK-52E cells with a DJ-1 overexpression vector (pEX2-EGFP-DJ-1; DJ-1 group) or an empty vector plasmid as the negative control group (vector group). The protein expression of DJ-1 was significantly increased following transfection with the overexpressio plasmid, as compared with that in the vector group (Fig. 3).

Effects of DJ-1 overexpression and NAC on H/R injury in $N R K-52 E$ cells following exposure to $H G$. To examine the molecular mechanisms of action of DJ-1 under HG and H/R conditions, we also treated the cells with the antioxidant, NAC. Both DJ-1 overexpression and NAC had no significant effects 

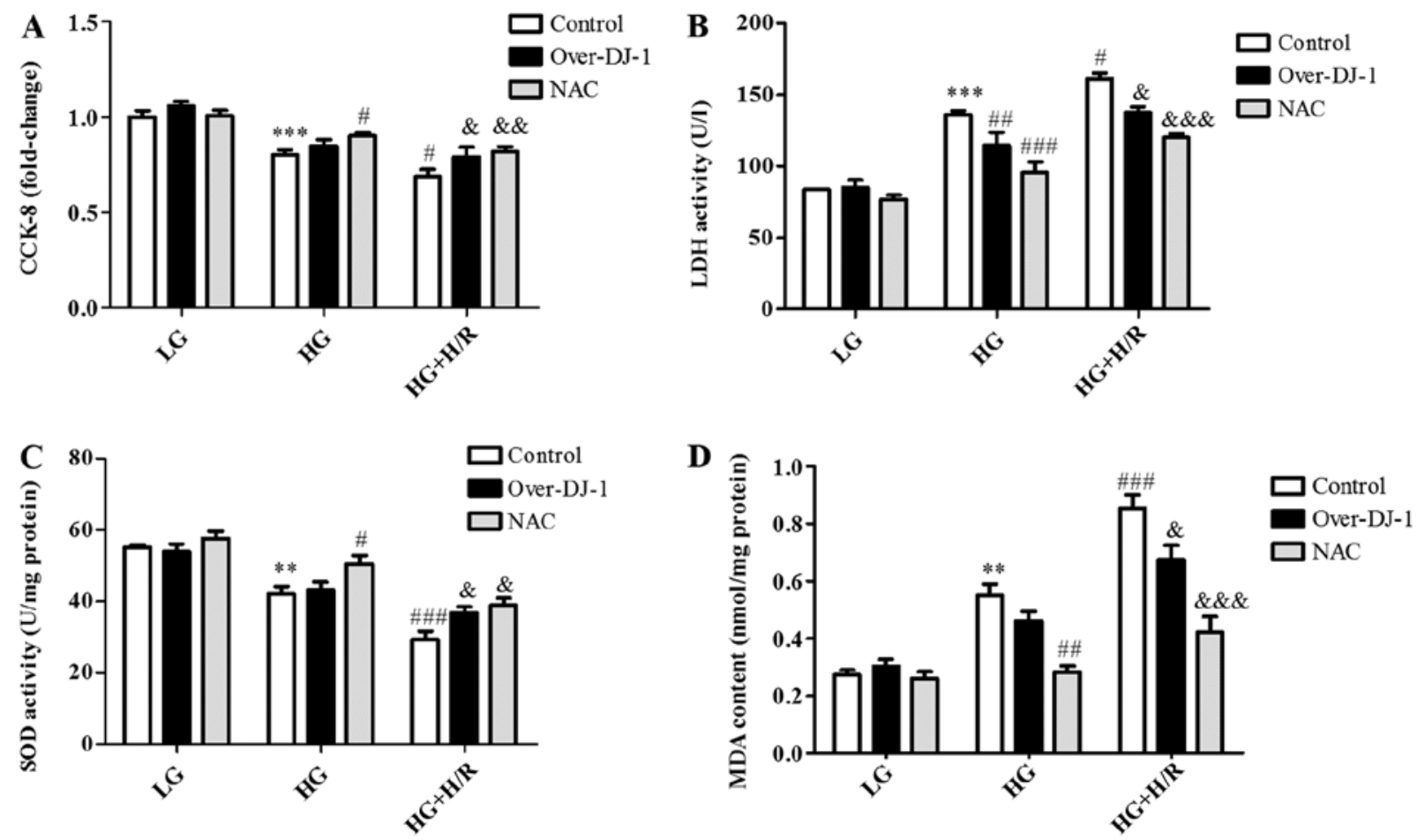

Figure 4. Effects of DJ-1 overexpression and NAC on H/R injury in NRK-52E cells following exposure to high glucose (HG). NRK-52E cells transfected with pEX-2-EGFP-DJ-1 or pre-treated of NAC were exposed to HG and hypoxia/reoxygenation (H/R) and we then measured (A) cell viability by CCK-8 assay; (B) LDH toxicity; (C) SOD activity; (D) the MDA content. Data are expressed as the means \pm SEM. ${ }^{* *} \mathrm{P}<0.01,{ }^{* * *} \mathrm{P}<0.001$ compared with the LG control group; ${ }^{\#} \mathrm{P}<0.05,{ }^{\# \#} \mathrm{P}<0.01,{ }^{\# \# \#} \mathrm{P}<0.001$ compared with the HG control group; ${ }^{\&} \mathrm{P}<0.05,{ }^{\text {\&\& }} \mathrm{P}<0.01$, \&\&\& $\mathrm{P}<0.001$ compared with the HG $+\mathrm{H} / \mathrm{R}$ control group. Results shown are representative of at least 3 independent experiments.

on CCK-8, LDH release, MDA content and SOD activity in the NRK-52 cells under LG conditions (Fig. 4). Exposure to HG significantly decreased the levels of CCK-8 and SOD activity, but significantly increased LDH release and the MDA content as compared with that in the LG group. All these changes were further intensified by H/R. However, DJ-1 overexpression and treatment with the antioxidant, NAC, significantly attenuated or reversed the increase in LDH release and the MDA content, and elevated the levels of CCK-8 and SOD activity.

Effects of DJ-1 overexpression and NAC on the protein expression of $\mathrm{Nrf} 2$ and $\mathrm{HO}-1$. After the NRK-52E cells were transfected with $\mathrm{pEX}$-2-EGFP-DJ-1, the protein expression levels of DJ-1, Nrf2 and HO-1 were significantly increased as compared with the corresponding control group transfected with the empty vector (Fig. 5A, C and E). In addition, treatment with NAC attenuated the HG-induced increase in DJ-1, Nrf2 and HO-1 expression, and attenuated the decrease in the protein expression of DJ-1 and Nrf2 following exposure to HG and H/R (Fig. 1B, D and F).

\section{Discussion}

The present observations provide several pieces of important evidence supporting the protective effects of DJ-1 against oxidative stress in response to $\mathrm{HG}$ and $\mathrm{H} / \mathrm{R}$ injury in renal cells. Firstly, it was demonstrated that HG decreased cell viability and SOD activity, but increased the MDA content, with DJ-1 protein expression increasing compensatively within $72 \mathrm{~h}$ of exposure to HG. Furthermore, H/R injury markedly decreased cell viability and increased oxidative stress in the cells exposed to HG, but decreased DJ-1 protein expressoin. Finally, the overexpression of DJ-1 exerted antioxidant effects against $\mathrm{HG}$ and $\mathrm{H} / \mathrm{R}$ injury, which were similar to the effects of NAC, promoting the protein expression of Nrf2 and HO-1. In short, the overexpression of DJ-1 reduced oxidative stress and attenuated H/R injury in NRK-52E cells exposed to HG.

It has been suggested that increased oxidative stress is recognized as the key factor in the pathogenesis and progression of DN (27-29). It has been indicated that HG enhances oxidative stress in renal cell injury (30). As an end-product of lipid peroxidation, MDA is widely used to detect the influence of oxidative stress on the mitochondria respiratory chain (31), while SOD is an antioxidant, protecting cells against oxidative stress. In the present study, the viability of NRK-2E cells was significantly decreased in a time-dependent manner following exposure to HG. A significant increase in MDA content and a decrease in SOD activity induced by HG were also observed. These results demonstrate that the antioxidant activity was weakened in parallel with the severity of NRK-52E cell injury. The results mentioned above indicate that the model of renal injury in oxidative stress was successfully established, which may be considered as an early-stage cell model of DN.

DJ-1 is an ubiquitous cytoprotective protein, and acts as an antioxidant to scavenge ROS in various cells. It has been shown that DJ-1 protects the morphology and function of the mitochondria and protects against cell injury (32). With the increase in blood glucose levels, the content of DJ-1 has been shown to increase in pancreatic $\beta$-cells, to inhibit the production of ROS (33). The antioxidant function of DJ-1 
A

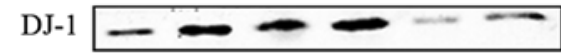

$\beta$-actin $=$

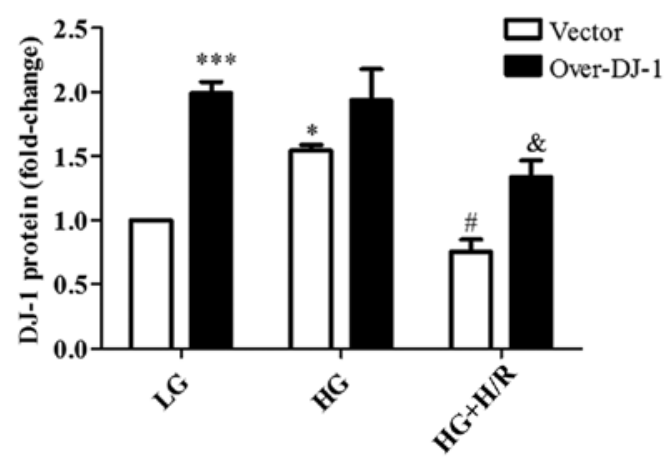

C
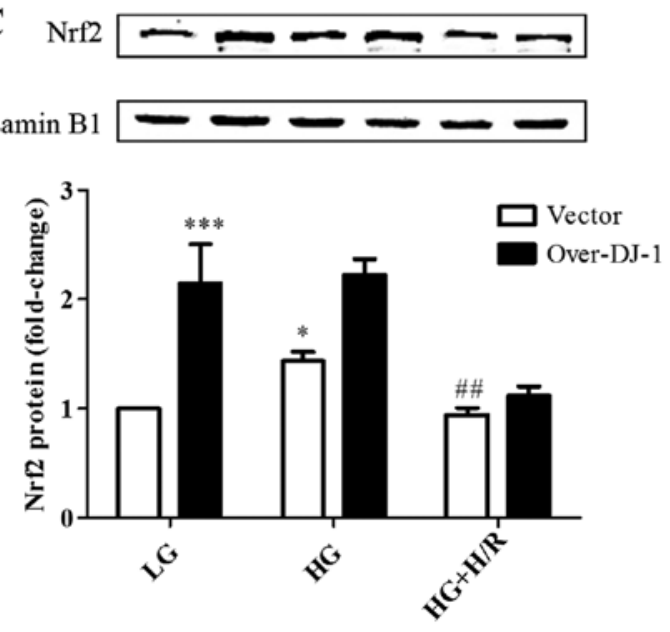

E

HO- 1
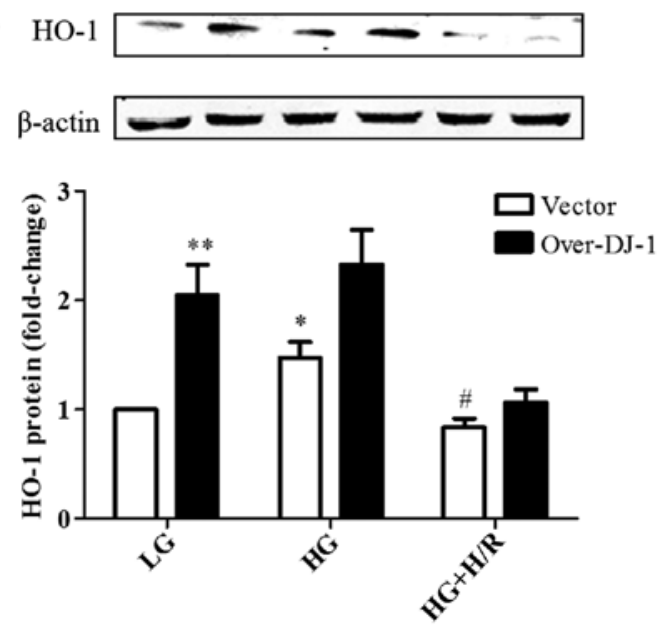

B
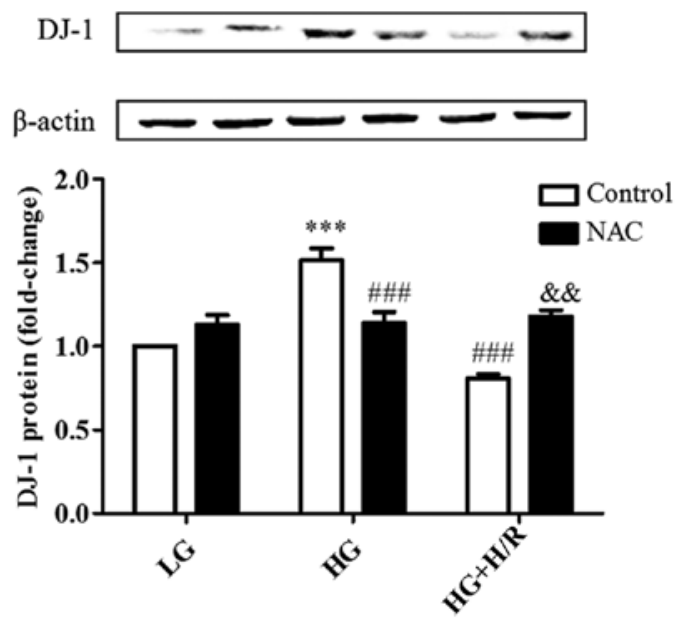

D
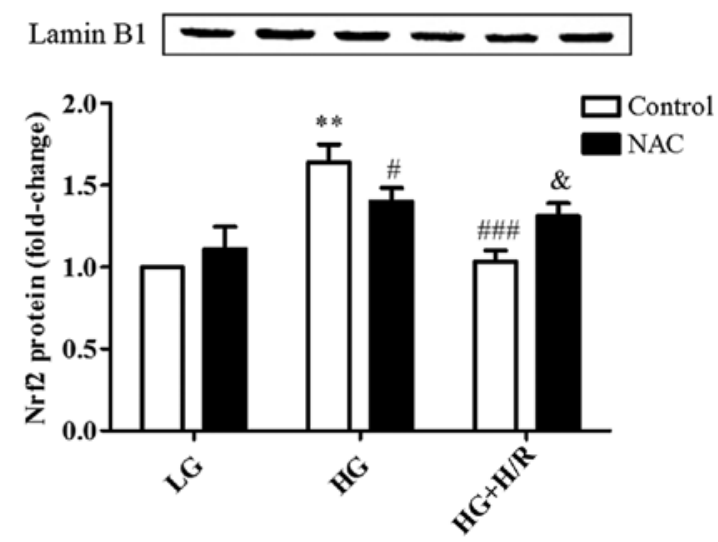

F
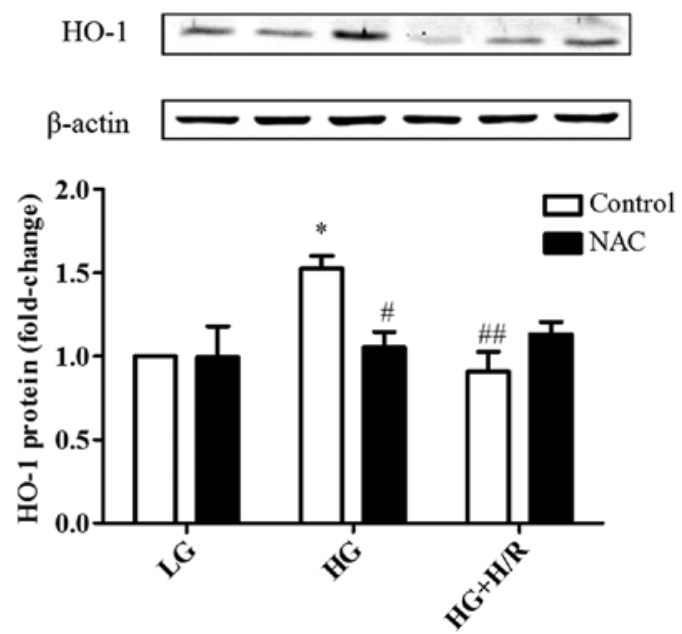

Figure 5. Effects of DJ-1 overexpression and NAC on the protein expression of nuclear factor (erythroid-derived 2)-like 2 (Nrf2) and heme oxygenase-1 (HO-1). (A, C and E) Western blot analysis results of DJ-1, Nrf2 and HO-1 protein expression in NRK-52E cells transfected with pEX-2-EGFP-DJ-1 and exposed to high glucose (HG) and hypoxia/reoxygenation (H/R); (B, D and F) western blot analysis results of DJ-1, Nrf2 and HO-1 protein in cells pre-treated with NAC and exposed to HG and H/R. $\beta$-actin was used for normalization of the total protein DJ-1 and HO-1 levels. Lamin B1 was used for normalization of the nuclear protein Nrf2. Data are expressed as the means \pm SEM. ${ }^{*} \mathrm{P}<0.05,{ }^{* *} \mathrm{P}<0.01,{ }^{* * *} \mathrm{P}<0.001$ compared with the $\mathrm{LG}$ vector (empty vector) control group; ${ }^{\#} \mathrm{P}<0.05,{ }^{\# \#} \mathrm{P}<0.01$,

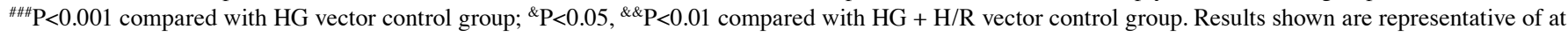
least 3 independent experiments.

maintains the integrity and physiological characteristics of the mitochondria, which is a prerequisite for glucose-stimulated insulin secretion. However, the mechanisms responsible for the protective effects of DJ-1 on renal tubular epithelial cells under HG conditions remain unclear. Our data demonstrated that the protein expression of DJ-1 was compensatively increased in a time-dependent manner and was significantly enhanced until the 72- $\mathrm{h}$ time period, but was decreased at the later time period of $96 \mathrm{~h}$ in the NRK-52E cells exposed to HG. Based on our results of the examination of cell injury and oxidative stress, 
we hypothesized that DJ-1 protein expression may be compensatively increased due to its antioxidant function in renal cells during the earlier time periods, but it is decreased following its depletion over a longer periods of time, and may thus be insufficient to protect against more severe oxidative stress. It can be considered that DJ-1 plays an important role in oxidative stress induced by HG in renal cells.

In the clinical peri-operative period, I/R injury occurs quite frequently, producing redundant ROS, leading to a multi-organ oxidative stress, including that in the kidneys (34). It has been shown that in rat renal I/R injury, the disrusption of mitochondrial metabolism and oxidative stress, results in ventricular function disorder, and leads to cardiac and renal injury (35). High glucose levels have been shown to highly associated with a a lower tolerance to ischemia, and an increased severity of renal I/R injury $(36,37)$. The present study demonstrated that the viability of renal cells in response to $H / R$ decreased, and the oxidative stress level increased, while the cells exposed to $\mathrm{HG}$ and $\mathrm{H} / \mathrm{R}$ exhibited more severe oxidative stress. It is inferred that in the peri-operative period, non-diabetic patients may suffer from oxidative stress injury induced by I/R; however, patients with diabetes may suffer more severely, and this matter requires more attention. We have already reported that cardiac DJ-1 expression was downregulated in hyperglycemiainduced I/R injury (20). However, there is little evidence of the association between I/R injury and DJ-1 in DN. In the present study, compared with the LG group, the NRK-52E cells in the $\mathrm{LG}+\mathrm{H} / \mathrm{R}$ group exhibited a much higher protein expression of DJ-1. However, compared with the $\mathrm{HG}$ group, the $\mathrm{HG}+\mathrm{H} / \mathrm{R}$ group exhibited a much lower protein expression of DJ-1. All the above-mentioned data indicated that $\mathrm{HG}$ or $\mathrm{H} / \mathrm{R}$ led to oxidative stress injury, which caused a compensatory increase in the protein expression of DJ-1 during the early stage. However, in the $\mathrm{HG}+\mathrm{H} / \mathrm{R}$ group, DJ-1 expression could not be maintained at higher, compensatory levels but was decreased, leading to more severe oxidative stress. This evidence proves DJ-1 to be vitally important for diabetic renal I/R injury in the peri-operative period.

Oxidative stress is involved in cellular injury in kidney, and overexpression of antioxidant proteins and treatment with antioxidants prevents renal damage. To further assess the cytoprotective function of DJ-1, we examined the overexpression of DJ-1 in H/R exposed to HG compared with the antioxidant NAC. Plasmid-derived overexpression of DJ-1 showed the antioxidative effect on high glucose and H/R injury. Interestingly, NAC played the similar role of DJ-1 overexpression in protecting cells against oxidative stress and increased DJ-1 expression during $\mathrm{H} / \mathrm{R}$ injury exposed to $\mathrm{HG}$. These results suggest that overexpression of DJ-1 can be partially explained by its inhibitory effects on oxidative stress induced by $\mathrm{HG}$ and $\mathrm{H} / \mathrm{R}$, yet weaker than NAC treatment.

Several enzymes and signaling pathways are related the antioxidative functions of DJ-1. It has been shown that renal I/R leads to increased apoptosis and oxidative stress in renal tubular epithelial cells, accompanied by the increased protein expression of Nrf2 and HO-1 (38). The activation of the DJ-1/Nrf2 pathway was found to exert a significant protective effect against oxidative stress $(21,39)$. The overexpression of DJ-1 reduces the ubiquitination of $\mathrm{Nrf} 2$ to stabilize the Nrf2 protein level, whereas the knockdown of DJ-1 decreases the stability of
Nrf2 protein (40). Our results revealed that the overexpression of DJ-1 promoted the expression of Nrf2 and HO-1 following exposure to HG and H/R injury. To gain insight into the potential mechanisms responsible for the protective effects of DJ-1 against H/R injury, we further evaluated the role of the DJ-1/ Nrf2 pathway by treating the cells with NAC. We observed a significant increase in the protein expression levels of DJ-1, $\mathrm{Nrf} 2$ and $\mathrm{HO}-1$ in the cells in the $\mathrm{HG}+\mathrm{H} / \mathrm{R}$ group treated with NAC. All the above-mentioned findings indicate that the upregulation of DJ-1 may attenuate the progression of diabetic renal I/R injury, and is associated with a significant increase in the expression levels of Nrf2 and HO-1.

In conclusion, the findings of the present study demonstrates that the overexpression of DJ-1 reduces oxidative stress and attenuates H/R injury in NRK-52E rat proximal tubular epithelial cells exposed to $\mathrm{HG}$.

\section{Acknowledgements}

This study was supported by grants from the National Natural Science Foundation of China (nos. 81170768, 81300674 and 81501648). We would like to thank the Central Laboratory, Renmin Hospital of Wuhan University (Wuhan, Hubei, China) for their support of our study.

\section{References}

1. Radcliffe NJ, Seah JM, Clarke M, MacIsaac RJ, Jerums G and Ekinci EI: Clinical predictive factors in diabetic kidney disease progression. J Diabetes Investig: April 25, 2016 (Epub ahead of print).

2. Verma AK, Chandra S, Singh RG, Singh TB, Srivastava S and Srivastava R: Serum prolidase activity and oxidative stress in diabetic nephropathy and end stage renal disease: A correlative study with glucose and creatinine. Biochem Res Int 2014: 291458, 2014.

3. Li B, Liu S, Miao L and Cai L: Prevention of diabetic complications by activation of Nrf2: Diabetic cardiomyopathy and nephropathy. Exp Diabetes Res 2012: 216512, 2012.

4. László E, Kiss P, Horváth G, Szakály P, Tamás A and Reglődi D: The effects of pituitary adenylate cyclase activating polypeptide in renal ischemia/reperfusion. Acta Biol Hung 65: 369-378, 2014.

5. Tawfik MK: Renoprotective activity of telmisartan versus pioglitazone on ischemia/reperfusion induced renal damage in diabetic rats. Eur Rev Med Pharmacol Sci 16: 600-609, 2012.

6. Nagakubo D, Taira T, Kitaura H, Ikeda M, Tamai K, Iguchi-Ariga SM and Ariga H: DJ-1, a novel oncogene which transforms mouse NIH3T3 cells in cooperation with ras. Biochem Biophys Res Commun 231: 509-513, 1997.

7. Aleyasin H, Rousseaux MW, Marcogliese PC, Hewitt SJ, Irrcher I, Joselin AP, Parsanejad M, Kim RH, Rizzu P, Callaghan SM, et al: DJ-1 protects the nigrostriatal axis from the neurotoxin MPTP by modulation of the AKT pathway. Proc Natl Acad Sci USA 107: 3186-3191, 2010.

8. Kim RH, Peters M, Jang Y, Shi W, Pintilie M, Fletcher GC, DeLuca C, Liepa J, Zhou L, Snow B, et al: DJ-1, a novel regulator of the tumor suppressor PTEN. Cancer Cell 7: 263-273, 2005.

9. Wilson MA: The role of cysteine oxidation in DJ-1 function and dysfunction. Antioxid Redox Signal 15: 111-122, 2011.

10. Billia F, Hauck L, Grothe D, Konecny F, Rao V, Kim RH and Mak TW: Parkinson-susceptibility gene DJ-1/PARK7 protects the murine heart from oxidative damage in vivo. Proc Natl Acad Sci USA 110: 6085-6090, 2013.

11. Taira T, Saito Y, Niki T, Iguchi-Ariga SM, Takahashi K and Ariga H: DJ-1 has a role in antioxidative stress to prevent cell death. EMBO Rep 5: 213-218, 2004.

12. Cuevas S, Yang Y, Konkalmatt P, Asico LD, Feranil J, Jones J, Villar VA, Armando I and Jose PA: Role of nuclear factor erythroid 2-related factor 2 in the oxidative stress-dependent hypertension associated with the depletion of DJ-1. Hypertension 65: 1251-1257, 2015. 
13. Cuevas S, Zhang Y, Yang Y, Escano C, Asico L, Jones JE, Armando I and Jose PA: Role of renal DJ-1 in the pathogenesis of hypertension associated with increased reactive oxygen species production. Hypertension 59: 446-452, 2012.

14. Moraes R, Valente RH, Leon IR, Trugilho MR, Pacheco AG, Nobrega AC, Gomes MB, Perales J and Tibirica E: Alterations of the kidney cortex proteome in response to exercise training in normoglycemic and hyperglycemic conditions. Curr Top Med Chem 14: 450-461, 2014.

15. Eltoweissy M, Dihazi GH, Müller GA, Asif AR and Dihazi H: Protein DJ-1 and its anti-oxidative stress function play an important role in renal cell mediated response to profibrotic agents. Mol Biosyst 12: 1842-1859, 2016.

16. Sun Q, Shen ZY, Meng QT, Liu HZ, Duan WN and Xia ZY: The role of DJ-1/Nrf2 pathway in the pathogenesis of diabetic nephropathy in rats. Ren Fail 38: 294-304, 2016.

17. Merikallio H, Pääkkö P, Kinnula VL, Harju T and Soini Y: Nuclear factor erythroid-derived 2-like $2(\mathrm{Nrf2})$ and DJ1 are prognostic factors in lung cancer. Hum Pathol 43: 577-584, 2012.

18. Kim W, Kim DW, Jeong HJ, Yoo DY, Jung HY, Nam SM, Kim JH, Choi JH, Won MH, Yoon YS, et al: Tat-DJ-1 protects neurons from ischemic damage in the ventral horn of rabbit spinal cord via increasing antioxidant levels. Neurochem Res 39: 187-193, 2014.

19. Yu HH, Xu Q, Chen HP, Wang S, Huang XS, Huang QR and He M: Stable overexpression of DJ-1 protects H9c2 cells against oxidative stress under a hypoxia condition. Cell Biochem Funct 31: 643-651, 2013.

20. Liu M, Zhou B, Xia ZY, Zhao B, Lei SQ, Yang QJ, Xue R, Leng Y, $\mathrm{Xu}$ JJ and Xia Z: Hyperglycemia-induced inhibition of DJ-1 expression compromised the effectiveness of ischemic postconditioning cardioprotection in rats. Oxid Med Cell Longev 2013. $564902,2013$.

21. Clements CM, McNally RS, Conti BJ, Mak TW and Ting JP: DJ-1, a cancer- and Parkinson's disease-associated protein stabilizes the antioxidant transcriptional master regulator Nrf2. Proc Natl Acad Sci USA 103: 15091-15096, 2006.

22. Jung KA, Choi BH, Nam CW, Song M, Kim ST, Lee JY and Kwak MK: Identification of aldo-keto reductases as NRF2-target marker genes in human cells. Toxicol Lett 218: 39-49, 2013

23. Lev N, Barhum Y, Ben-Zur T, Melamed E, Steiner I and Offen D: Knocking out DJ-1 attenuates astrocytes neuroprotection against 6-hydroxydopamine toxicity. J Mol Neurosci 50: 542-550, 2013.

24. Zhang L, Zhu Z, Liu J, Zhu Z and Hu Z: Protective effect of $\mathrm{N}$-acetylcysteine (NAC) on renal ischemia/reperfusion injury through Nrf2 signaling pathway. J Recept Signal Transduct Res 34: 396-400, 2014.

25. Fouad AA, Al-Mulhim AS, Jresat I and Morsy MA: Protective effects of captopril in diabetic rats exposed to ischemia/reperfusion renal injury. J Pharm Pharmacol 65: 243-252, 2013.

26. Zhou SP, Liao WT, Yang LK and Sun L: Effects of sevoflurane pretreatment on renal Src and FAK expression in diabetic rats after renal ischemia/reperfusion injury. Mol Cell Biochem 384 203-211, 2013
27. Forbes JM, Coughlan MT and Cooper ME: Oxidative stress as a major culprit in kidney disease in diabetes. Diabetes 57: 1446-1454, 2008

28. Giacco F and Brownlee M: Oxidative stress and diabetic complications. Circ Res 107: 1058-1070, 2010.

29. Yamagishi S and Matsui T: Advanced glycation end products, oxidative stress and diabetic nephropathy. Oxid Med Cell Longev 3: 101-108, 2010.

30. Zhang X, Zhao Y, Chu Q, Wang ZY, Li H and Chi ZH: Zinc modulates high glucose-induced apoptosis by suppressing oxidative stress in renal tubular epithelial cells. Biol Trace Elem Res 158: 259-267, 2014.

31. Gutteridge JM and Halliwell B: The measurement and mechanism of lipid peroxidation in biological systems. Trends Biochem Sci 15: 129-135, 1990.

32. Lin TK, Liou CW, Chen SD, Chuang YC, Tiao MM, Wang PW, Chen JB and Chuang JH: Mitochondrial dysfunction and biogenesis in the pathogenesis of Parkinson's disease. Chang Gung Med J 32: 589-599, 2009.

33. Jain D, Jain R, Eberhard D, Eglinger J, Bugliani M, Piemonti L, MarchettiP and LammertE: Age- and diet-dependent requirement of DJ-1 for glucose homeostasis in mice with implications for human type 2 diabetes. J Mol Cell Biol 4: 221-230, 2012.

34. Szeto HH: Mitochondria-targeted cytoprotective peptides for ischemia-reperfusion injury. Antioxid Redox Signal 10: 601-619, 2008.

35. Tai ST, Fu YH, Yang YC and Wang JJ: Niacin ameliorates kidney warm ischemia and reperfusion injury-induced ventricular dysfunction and oxidative stress and disturbance in mitochondrial metabolism in rats. Transplant Proc 47: 1079-1082, 2015.

36. Caetano AM, Vianna Filho PT, Castiglia YM, Golim MA, de Souza AV, de Carvalho LR, Deffune E, de Oliveira C and Vianna PT: Erythropoietin attenuates apoptosis after ischemiareperfusion-induced renal injury in transiently hyperglycemic Wister rats. Transplant Proc 43: 3618-3621, 2011.

37. de Souza AV, Golim MA, Deffune E, Domingues MA, de Carvalho LR, Vianna IG, Castiglia YM and Vianna PT: Evaluation of renal protection from high doses of melatonin in an experimental model of renal ischemia and reperfusion in hyperglycemic rats. Transplant Proc 46: 1591-1593, 2014.

38. Jiang G, Liu X, Wang M, Chen H, Chen Z and Qiu T: Oxymatrine ameliorates renal ischemia-reperfusion injury from oxidative stress through Nrf2/HO-1 pathway. Acta Cir Bras 30: 422-429, 2015.

39. Bahmed K, Messier EM, Zhou W, Tuder RM, Freed CR, Chu HW, Kelsen SG, Bowler RP, Mason RJ and Kosmider B: DJ-1 modulates Nrf2-mediated protection in human primary alveolar type II cells in smokers. Am J Respir Cell Mol Biol: April 19, 2016 (Epub ahead of print)

40. Gan L, Johnson DA and Johnson JA: Keap1-Nrf2 activation in the presence and absence of DJ-1. Eur J Neurosci 31: 967-977, 2010. 